\title{
Improvement in image quality in obese patients by using a new $x$-ray tube and Deep Learning Image Reconstruction in Coronary Computed Tomography Angiography
}

Anne-Sofie Horsted Brunebjerg ( $\square$ annehorsted@gmail.com )

Svendborg Hospital: Svendborg Sygehus https://orcid.org/0000-0001-6884-5331

\section{Svea Deppe Mørup}

University College Lillebaelt: UCL Erhvervsakademi og Professionshojskole

Thomas Rueskov Andersen

Svendborg Hospital: Svendborg Sygehus

Roda Abdulkadir Mohamed

Svendborg Hospital: Svendborg Sygehus

Jess Lambrechtsen

Svendborg Hospital: Svendborg Sygehus

\section{Research Article}

Keywords: Coronary computed tomography angiography (CCTA), coronary artery disease (CAD), deep learning image reconstruction (DLIR), image quality, diagnostic utility.

Posted Date: February 24th, 2022

DOI: https://doi.org/10.21203/rs.3.rs-1384940/v1

License: (c) (i) This work is licensed under a Creative Commons Attribution 4.0 International License.

Read Full License 


\section{Introduction}

Ischemic heart disease is one of the leading causes of death and hospitalization [1]. The gold standard method in diagnosing stable coronary artery disease (CAD) is invasive coronary angiography (ICA). This is, however, an invasive procedure and therefore associated with risks of peri-procedural complications [2]. Coronary computed tomography angiography (CCTA) is an established non-invasive image modality for a rapid and accurate evaluation of the coronary arteries in patients with low- to intermediate-risk of stable CAD [3, 4]. However, it is a challenge to obtain diagnostic image quality in obese patients due to increased noise from the excessive adipose tissue $[3,5,6]$. According to the World Health Organization (WHO), more than 650 million adults worldwide suffer from obesity and the number is rising [7]. This represents a diagnostic challenge, since obesity is also associated with increased risk of CAD thus making clear visualization and evaluation of coronary arteries important in this particular group of patients $[6,8]$.

Different techniques have been proposed to increase image quality in obese patients. This includes a higher tube current and an increase in tube potential which, theoretically, should improve image quality by reducing noise [9]. In this study, a new x-ray tube (Quantix 160 from GE Healthcare, Waukesha, WI, USA) has upgraded the Revolution CT scanner to a Revolution Apex (GE Healthcare, Waukesha, WI, USA) which has enabled us to increase tube current up to $1300 \mathrm{~mA}$ for some $\mathrm{kV}$ settings [10]. The effect of higher tube currents is to increase the amount of the $\mathrm{x}$-rays and to increase the signal reaching the detectors and hereby improve image quality.

Another promising technique in CT image reconstruction is the use of deep learning image reconstruction (DLIR) [11]. TrueFidelity (TrueFidelity, GE Healthcare, Waukesha, WI, USA) is a new type of DLIR that has been developed to reduce noise and improve image quality without the need for a higher radiation dose $[10,12,13]$. The study from Solomon, J. et al. has tested DLIR on phantoms and found reduced noise with the use of DLIR compared to conventional filtered back projection (FBP) [12]. Furthermore, Benz et al. have tested the diagnostic utility of DLIR in a clinical setting and compared it to adaptive statistical iterative reconstruction-Veo (ASiR-V) and likewise found that DLIR significantly reduces noise in CCTA [11]. However, no studies have tested the combined effect of DLIR and the Revolution Apex in obese and very obese patients.

Therefore, the aim of this study is to evaluate possible improvements in image quality, diagnostic utility and noise reduction in symptomatic obese and very obese patients with low- to intermediate-risk of stable CAD by using the Revolution Apex scanner and DLIR, respectively. 


\section{Materials and Methods}

\section{Patient population}

The study included 72 patients referred to a CCTA because of mild to moderate symptoms of CAD between December 2018 and July 2019. Out of these, 51 patients underwent CCTA twice. The follow-up scans were performed with the Revolution Apex scanner between October 2020 and November 2020. Originally, the followup was planned to be carried out approximately 6 months after the first examination, but delivery issues with the new tube and the Covid-19 pandemic delayed the study. Patients were stratified by Body Mass Index (BMI) into two groups; 1) 21 patients with BMI 30-34,9 kg/m² in the "obese" group and 2) 30 patients with BMI $\geq 35 \mathrm{~kg} / \mathrm{m}^{2}$ in the "very obese" group. The trial was approved by the local ethics committee, and informed consent was obtained from all patients. The inclusion criteria were: age $>18$ years, GFR $>45 \mathrm{ml} / \mathrm{min}$, heart rate (HR) between 50-85 beats/min (BPM). Exclusion criteria were: irregular heart rhythm, previous percutaneous coronary intervention (PCI), history of allergic reactions to contrast agents, and patients who had experienced significant cardiac events since the baseline CCTA. Significant cardiac events were defined as PCI or coronary artery bypass grafting $(\mathrm{CABG})$.

On the day of the baseline CCTA, a patient examination and a structured interview provided information regarding risk factors: 1) BMI (height and weight), 2) blood pressure (BP), 3) smoking history (active, previous or nonsmoking), 4) diabetes mellitus (treatment with insulin, oral hypoglycemic agents, or non-pharmacological treatment), 5) family history of CAD, 6) treatment with anti-hypertensive drugs, and 7) treatment with lipidlowering drugs. Furthermore, the indication of the CCTA was retrospectively obtained from each patient's hospital record, and if "angina" was the indication, it was further specified (typical angina, atypical angina, unspecific chest pain, dyspnea, or other reasons).

On the day of the follow-up CCTA, performed with the Revolution Apex scanner, the patients were interviewed regarding any significant changes in health status since their first CCTA. Significant changes were defined as: Acute myocardial infarction (AMI), PCI, CABG, newly developed diabetes, and change in anti-hypertensive or lipid-lowering treatment. In addition, a new BMI was calculated.

\section{Image acquisition}

The baseline CCTA examinations were performed on a 256-slice scanner (Revolution CT from GE Healthcare, Waukesha, WI, USA) with a gantry rotation time of $280 \mathrm{~ms}$. The tube voltages used were 100,120 , or $140 \mathrm{kV}$, which was determined individually by the radiographers by evaluating each patient's size. The automatic tube 
current modulation was set between 100 and $750 \mathrm{~mA}$, and the scanner's automatic tube current modulation (ATCM) selected the $\mathrm{mA}$ for all patients between $328-739 \mathrm{~mA}$, depending on patient size, and muscle and adipose tissue distribution.

The follow-up CCTA examinations were performed on the 256-slice CT scanner upgraded to the Revolution Apex with the new x-ray tube Quantix. The gantry rotation time remained unchanged at $280 \mathrm{~ms}$, and the tube voltage was matched individually to each patient's tube voltage used in the baseline CCTA and therefore remained unchanged as well. The revolution Apex scanner comes with an automatic tube voltage selection [kV-assist 2.0] that makes it possible for the system to select the tube voltage. However, this feature was not used in our study, as we wanted a direct comparison between the two CCTAs. Therefore, the tube voltage was set manually while the ATCM automatically selected the tube current between 500 and $1300 \mathrm{~mA}$ (533-1080).

All patients were prescribed $2 \times 7,5 \mathrm{mg}$ tablet of Ivabradine, 1 tablet the night before the scan and 1 tablet in the morning of the CCTA. Moreover, all patients received 1 dose of sublingual nitroglycerin spray $(0,4 \mathrm{mg} / \mathrm{dose}) 2-5$ minutes before each CCTA in order to dilate the coronary arteries. HR was determined right before the scan and, in case of a HR > 60 BPM, IV Metoprolol was given to lower the HR. Iodixanol contrast, Visipaque (GE Healthcare, Buckinghamshire, UK) with a iodine content of $320 \mathrm{mg}$ iodine $/ \mathrm{ml}$, was injected in a dose of 60-70 ml with an injection flow rate of $6-7 \mathrm{ml} / \mathrm{sec}$.

\section{Image reconstruction}

All images were reconstructed with a standard kernel, a slice thickness of $0,625 \mathrm{~mm}$, and 50\% ASiR-V on the Revolution CT scanner. All scans performed on the Revolution Apex scanner were reconstructed with 50\% ASiRV as well as with DLIR TrueFidelity "High".

\section{$\underline{\text { Subjective image quality assessment }}$}

Image analysis was performed retrospectively, and all image sets were assessed by a Level III CCTA certified cardiologist. Image quality was evaluated according to each of the quality criteria listed in Table 1 . The assessment of criteria 1-6 was performed according to the 5-point Likert scale which grades each of the criteria into one of the 5 categories listed in Table 1 [14]. Additionally, the observer was asked to evaluate the diagnostic utility as either "diagnostic" or "non-diagnostic". If the image sets were assessed as "non-diagnostic", the observer selected between the following reasons for the artifact(s): A: motions, B: noise, C: IV contrast, and/or D: calcium. 
All image sets were randomized in ViewDEX 2.0 [15] and visually assessed one at a time by the observer who was blinded to clinical information, date of scan, and technical data. Only the standard series at the $75 \%$ phase was shown, and no further analysis was allowed for interpretation.

\section{$\underline{\text { Noise measurements }}$}

Two independent reviewers placed regions of interest (ROI) in both the old ASIR-V, the new ASIR-V, and in the DLIR images. Two ROIs were placed, 1) in the middle of the lumen of aorta (ROIAo), and 2) in the interventricular septum in the myocardium (ROIMyo). The ROIs in the aortic lumen were identical at $20 \times 20 \mathrm{~mm}$, while the ROIs in the myocardium were modified based on anatomy. All ROIs were placed in axial plane reconstruction, and the mean Hounsfield unit (HU) value and corresponding noise measurement (standard deviation (SD)) value was recorded at each ROI.

\section{$\underline{\text { Statistical analyses }}$}

The patient characteristics at baseline and follow-up, presented in Table 2, were calculated as means \pm standard deviations (SD) or simply presented as numbers $[n]$ and as percent of the total study population. Missing data were omitted, and in the specific case of the coronary artery calcium score (CAC), only patients with a known value in both the baseline and the follow-up scan were included in order to assure direct patient-to-patient comparison. Comparisons between the groups were performed using a paired sample $t$-test.

The analyses of the subjective image quality were performed using an ordered logistic regression [proportional odds model] with the Likert scores [1-5] stratified by the CT scanner and reconstruction type (Revolution CT + ASiR-V, Revolution Apex + ASiR-V, and Revolution Apex + DLIR), and odds ratios (OR) were given. The same analysis model was performed with the "overall image quality" that was defined as the total sum of the Likert score from quality criteria 1-6. The diagnostic utility was calculated as the percentage of images assessed as "diagnostic" and "non-diagnostic", respectively, for each of the three CT acquisition groups.

The analyses of the noise measurements were performed using linear regression with the ROI noise-measurements ( $\mathrm{ROI}_{\mathrm{Ao}}$ and $\mathrm{ROI}_{\mathrm{Myo}}$ ) with the "Revolution $C T+A S i R-V$ " and "Revolution Apex + DLIR" as dependent variables and "Revolution Apex $+A S i R-V$ " as the independent variable, and $\square$-coefficients were calculated.

All statistical analyses were performed using C Stata/IC 16.0 (StataCorp LLC, College Station, TX, USA). A pvalue of less than 0,05 was considered statistically significant. 


\section{Results}

\section{$\underline{\text { Patient characteristics and scan parameters }}$}

Initially, a total of 72 patients met the inclusion criteria at the baseline scan and were included in the study. Of these, 21 patients were cut out afterwards due to the following exclusion criteria: irregular heart rhythm, HR $>85$ BPM, PCI between baseline and follow-up scan, technical issues with retrieving the correct image series needed for comparison, and, finally, some patients did either not show up or did no longer wish to participate in the second CCTA, partly due to the Covid-19 pandemic. Hence, the final study population consisted of 51 patients. In the time between the first and the second CCTA, 4 patients had changes in their cardiovascular pharmacological treatment, 1 patient had undergone a balloon angioplasty, and 1 patient had recently been diagnosed with type 2 diabetes. Furthermore, 5 patients had gained, and 3 patients had lost, more than $10 \mathrm{~kg}$. Patient and CT acquisition characteristics are shown in Table 2.

In average, the patients received a 18,35\% higher radiation dose with the Revolution Apex scanner (mean DLP = $187 \mathrm{mGy} * \mathrm{~cm}$ ) compared to the Revolution CT (mean DLP $=158 \mathrm{mGy} * \mathrm{~cm})$.

\section{Image quality and diagnostic utility}

An overview of the image quality assessment is presented in Table 3 . The overall image quality was significantly improved with Revolution Apex when images were reconstructed with DLIR $(p=0,017)$ compared to image sets performed with Revolution CT and conventional reconstruction with ASiR-V. For criterion 2) Visually sharp delineation of the vessel wall of the left anterior descending artery (LAD) $(p=0,001)$, criterion 5) Visualization of the myocardial septum between the right and left ventricle $(p=0,005)$, and criterion 6$)$ Homogeneity in the left/right ventricle $(p=0,009)$ significant image quality improvements were found for Revolution Apex with DLIR. In the same criteria 2, 5, 6, and 7, no significant differences were found with Revolution Apex and conventional reconstruction with ASiR-V. Regardless of the CT scanner and the reconstruction method, no significant differences were found in criteria 1,3 , and 4 (sharp/clear delineation of the aortic wall, visually sharp delineation of the vessel wall of RCA and CX). An example of the visual representation of the three different CCTA modalities from one of the patients is shown in Fig. 1.

The diagnostic utility of the image sets performed with Revolution CT and ASiR-V was similar to those performed with Revolution Apex and ASiR-V with 69\% of the images considered diagnostic and 31\% non-diagnostic. 
However, with the use of Revolution Apex and DLIR, the diagnostic utility was improved with $88 \%$ of the images assessed as diagnostic and $12 \%$ as non-diagnostic. The reasons for the artifacts in non-diagnostic images are presented in Fig. 2. It appears that the most frequent chosen reason for artifacts was motion closely followed by noise, except for the images performed with Revolution Apex and DLIR, where only one of the artifacts in the image series was caused by noise.

\section{Noise}

The noise results are presented in Table 4. These results show a significant reduction in noise in the images performed with Revolution Apex and DLIR compared to Revolution Apex and ASiR-V. This was the case for both $\operatorname{ROI}_{\text {Ao }}(p=<0,001)$ and $\mathrm{ROIMyo}_{\text {Mo }}(p=0,015)$. However, there were no significant changes in noise when using the Revolution Apex and ASiR-V compared to Revolution CT and ASiR-V.

\section{Discussion}

The study demonstrates that, compared to the Revolution CT and ASiR-V reconstruction, the Revolution Apex in combination with DLIR significantly increases image quality, reduces noise, and raises diagnostic utility from $69 \%$ to $88 \%$ in obese and very obese patients. When eliminating DLIR and looking at the new Quantix $160 \mathrm{x}$-ray tube alone with conventional ASiR-V, no statistical improvements have been detected - not in image quality, nor in diagnostic utility or noise reduction. The patients received an increased radiation dose with the Revolution Apex scanner compared to the Revolution CT scanner, and this suggests that the Revolution CT scanner had reached its limits regarding maximum tube current and radiation dose in patients of this size.

One of the hypotheses of this study was that the combined effect of the Revolution Apex with DLIR would result in significantly improved image quality. The study found improvements in image quality and an average noise reduction of $41 \%$. This finding is consistent with the study from Benz et al. (2020), who have found a substantial reduction in image noise of up to $43 \%$ and an increase in image quality of up to $138 \%$ when reconstructing with DLIR compared to ASiR-V [11]. Another important finding of the present study is the increased diagnostic value of DLIR with $88 \%$ of the image sets performed with DLIR being approved for diagnostic use compared to only $69 \%$ with ASiR-V. This result differs from that of Benz et al. (2020) who found the diagnostic accuracy of DLIR to be equal to that of ASiR-V. However, the definition of "diagnostic approval" in our study is limited to a subjective assessment while Benz et al. have tested the diagnostic accuracy by comparing the CCTA diagnoses with ICA. Our study is based on a larger study population, and the results could imply a greater diagnostic utility 
with the use of DLIR in obese and very obese patients, but further studies are needed to support this finding. Moreover, Benz et al. only included patients with a high burden of CAD, which makes the generalizability of their findings limited to this particular patient group. This could pose an issue since the use of CCTA as a stand-alone diagnostic tool is more frequent in patients with low- to intermediate-risk of CAD. The present study examines this particular group of patients with low- to intermediate-risk of CAD and is the first clinical trial to test DLIR in obese and very obese patients. Hereby, it contributes to filling an important gap in our present knowledge. With these results, it is fair to suggest that some of the results that Benz et al. found in high-risk patients regarding improved image quality also apply to low- to intermediate-risk patients.

When analyzing the quality criteria individually, the improvement in image quality is seen in both criteria 5 and 6 regarding noise, while only one of the four criteria regarding spatial resolution, criteria 2, is significantly improved. This implicates, that the positive effect of DLIR on image quality can mainly be ascribed to the noise reduction and less to the spatial resolution. This finding is consistent with that of Solomon et al. (2020), who have tested DLIR on different phantoms, and found that, compared to FBP, DLIR demonstrates a strong noise magnitude reduction, while noise texture and high-contrast spatial resolution remain unchanged [12]. Even though the study designs of these two studies have some important differences, the findings illustrate and support the same strengths and weaknesses of DLIR. This finding is further supported by the reasons for the artifacts in non-diagnostic images that are presented in Fig. 2. These are showing a decrease in the number of images being assessed as non-diagnostic due to noise. This supports the result that DLIR reduces noise and indicates that the noise-reduction performed by DLIR has an impact on the diagnostic utility.

Contrary to expectations, the results of this study did not show any significant improvements in the image sets performed with Revolution Apex and conventional reconstruction with ASiR-V. Given the characteristics of the study population, which consists of only obese and very obese patients, it was hypothesized that an increase in tube current and radiation dose should result in improved image quality and noise reduction [9]. As seen in Table 2 , both the tube current and the radiation dose have been increased as intended, but without any significant effect on image quality and noise levels. These results could imply that the increase in tube current does not affect the visual image quality in obese or very obese patients when reconstructed with iterative reconstruction. However, another possible explanation could be the inclusion of both obese and very obese patients. Theoretically, image quality should decrease with increasing BMI due to increased noise from the excessive adipose tissue. Therefore, it could be interesting to perform a study that includes only the very obese patients and evaluate if the effect of increased tube current and radiation dose is better in this particular patient group. 
By increasing the tube current, the radiation dose was increased as well, but if this is ensued by an improvement in image quality for this particular patient group, the benefits should compensate for the risks of a higher radiation dose. However, in this study there was no significant benefit from increased radiation dose, and ,consequently, it is reasonable to conclude that more studies regarding this topic are needed before recommending increased radiation doses in clinical practice.

This study has some limitations. First, the time between the two CCTAs was intended to be no longer than 6 months but was extended due to the previously mentioned issues. This has resulted in a maximum duration between the first and the second CCTA for almost 22 months for some patients. However, as the measured parameter in this study is the subjective image quality, the possible changes in cardiac health (such as increased CAC) during this period of time should not impact the comparability between the two CCTAs. Furthermore, each patient was interviewed and examined on the day of the second scan in order to detect any changes that might affect the comparison potential of the two CCTAs, such as significant weight loss or gain. However, despite the individual changes in BMI observed in some of the patients, the two groups have shown to be similar. As seen in Table 2, the CAC values have increased from the first CCTA to the second CCTA, and the change in CAC is statistically significant. The CAC, however, is expected to increase over time and should not implicate differences in the image quality assessments between baseline and follow-up. A strength of this study is the direct comparison, as the patients are compared to themselves. Another strength is the number of participants in this study, which increases its power and makes it the largest study regarding DLIR until today.

The relatively high number of non-diagnostic CCTAs is, of course, due to the previously mentioned limitations when scanning obese patients. However, the restrictions for interpretation for the cardiologist of all available series of images in this study further rise the proportion of non-diagnostic scans considerably.

This study is based on obese and very obese patients with low- to intermediate-risk of CAD, which limits the generalizability of the results to this particular patient group. This group of patients, however, was chosen because of the diagnostic challenge that patients with a high BMI represents when performing CCTA, and the belief that the hypotheses tested in this study would benefit this group in particular. Future studies should examine other patient groups that represent a diagnostic challenge with the present CCTA tools e.g. patients with stents or patients with a lot of calcification. The DLIR used in this study, TrueFidelity, is available in both Low, Medium and High levels, but in this study only the High level was included. Therefore, it could be interesting for future studies to include both Medium and Low levels and analyze the potential effect on spatial resolution. 
In summary, reconstruction with DLIR in obese and very obese patients with a mild- to moderate-risk of CAD has improved the perceived image quality and diagnostic utility, and reduced noise levels, compared to reconstruction with ASiR-V. The replacement of the previous x-ray tube with the new Quantix 160 and the increase in tube current did not result in any significant differences when reconstructing the image sets with the same reconstruction method (ASiR-V). More studies regarding both DLIR and the Quantix 160 x-ray tube are still needed.

\section{Declarations}

\section{$\underline{\text { Conflicts of interest }}$}

Nothing to disclose.

\section{Consent to participate}

Informed consent was obtained from all individual participants included in the study. 


\section{References}

1. World Health Organisation. The top 10 causes of death 2020 [cited 2020 Dec]. Available from: https://www.who.int/news-room/fact-sheets/detail/the-top-10-causes-ofdeath.

2. Segev OL, Gaspar T, Halon DA, Peled N, Domachevsky L, Lewis BS, et al. Image quality in obese patients undergoing 256-row computed tomography coronary angiography. Int J Cardiovasc Imaging. 2012;28[3]:633-9.

3. Zimmerman SL, Kral BG, Fishman EK. Diagnostic quality of dual-source coronary CT examinations performed without heart rate control: importance of obesity and heart rate on image quality. J Comput Assist Tomogr. 2014;38[6]:949-55.

4. Montalescot G, Sechtem U, Achenbach S, Andreotti F, Arden C, Budaj A, et al. 2013 ESC guidelines on the management of stable coronary artery disease: the Task Force on the management of stable coronary artery disease of the European Society of Cardiology. Eur Heart J. 2013;34[38]:2949-3003.

5. Ghekiere O, Salgado R, Buls N, Leiner T, Mancini I, Vanhoenacker P, et al. Image quality in coronary CT angiography: challenges and technical solutions. Br J Radiol. 2017;90[1072]:20160567.

6. Lee AM, Engel LC, Hui GC, Liew G, Ferencik M, Sidhu MS, et al. Coronary computed tomography angiography at $140 \mathrm{kV}$ versus $120 \mathrm{kV}$ : assessment of image quality and radiation exposure in overweight and moderately obese patients. Acta Radiol. 2014;55[5]:554-62.

7. World Health Organisation. Obesity and overweight 2020. Available from: https://www.who.int/news-room/fact-sheets/detail/obesity-and-overweight.

8. $\quad$ Lu Y, Hajifathalian K, Ezzati M, Woodward M, Rimm EB, Danaei G. Metabolic mediators of the effects of body-mass index, overweight, and obesity on coronary heart disease and stroke: a pooled analysis of 97 prospective cohorts with 1.8 million participants. Lancet. 2014;383[9921]:970-83.

9. Seeram E. Computed Tomography : Physical Principles, Clinical Applications, and Quality Control. 4th ed: Elsevier Inc.; 2016.

10. Thibault J, Utschig, M. Revolution Apex with QuantixTM 160 [White paper]. 2020 .

11. Benz DC, Benetos G, Rampidis G, von Felten E, Bakula A, Sustar A, et al. Validation of deep-learning image reconstruction for coronary computed tomography angiography: Impact on noise, image quality and diagnostic accuracy. J Cardiovasc Comput Tomogr. 2020;14[5]:444-51.

12. Solomon J, Lyu P, Marin D, Samei E. Noise and spatial resolution properties of a commercially available deep learning-based CT reconstruction algorithm. Med Phys. 2020;47[9]:3961-71.

13. Jiang H LE, Nett B, Tang J, Thibault J, Sahney S. A new era of image reconstruction: TrueFidelityTM Technical white paper on deep learning image reconstruction [White paper]. 2020.

14. Seppelt D, Kolb C, Kühn JP, Speiser U, Radosa CG, Hoberück S, et al. Comparison of sequential and high-pitch-spiral coronary CT-angiography: image quality and radiation exposure. Int J Cardiovasc Imaging. 2019;35[7]:1379-86.

15. Håkansson M, Svensson S, Zachrisson S, Svalkvist A, Båth M, Månsson LG. VIEWDEX: an efficient and easy-to-use software for observer performance studies. Radiat Prot Dosimetry. 2010;139[1-3]:42-51. 

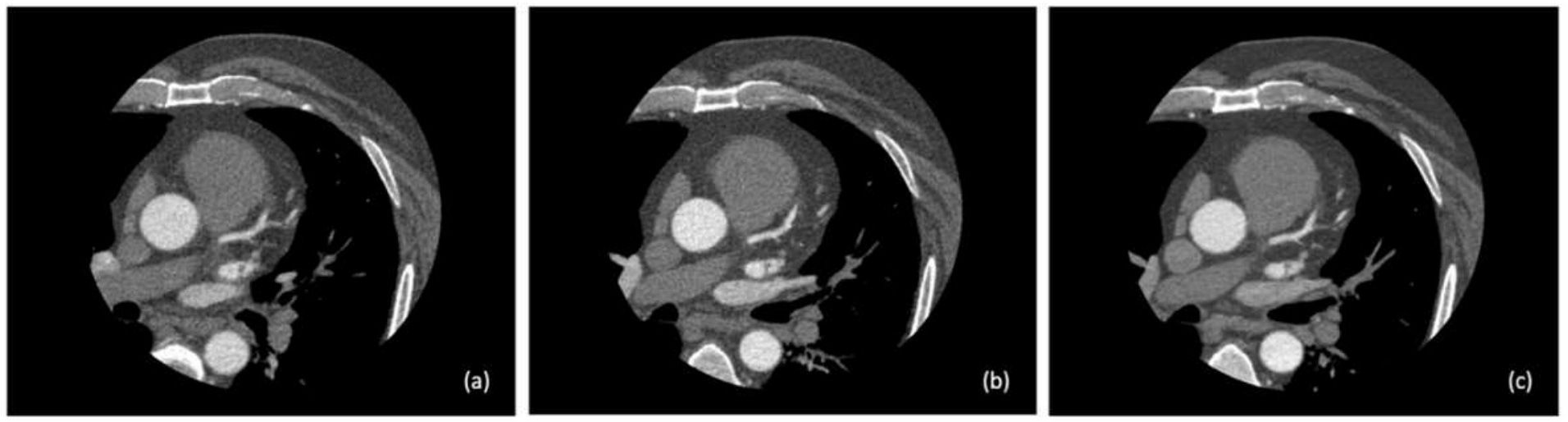

\section{Figure 1}

CCTA images, from the same patient, illustrating (a) image performed with Revolution CT and reconstructed with ASiR-V, (b) image performed with Revolution Apex and reconstructed with $A S i R-V$, and (c) image performed with Revolution Apex and reconstructed with DLIR.

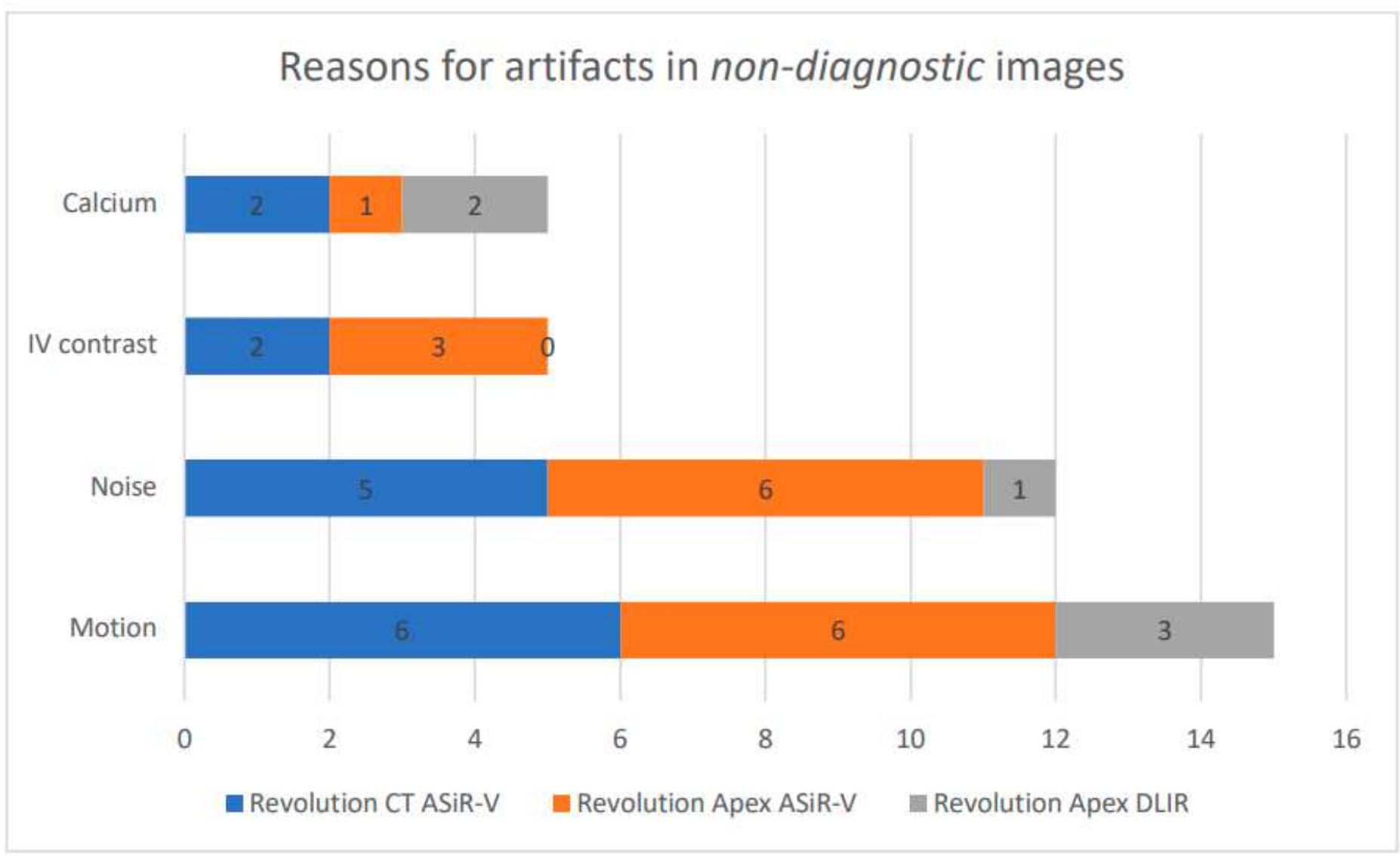

Figure 2

The selected reasons for artifacts in images assessed as "non-diagnostic". 


\section{Supplementary Files}

This is a list of supplementary files associated with this preprint. Click to download.

- Table1.pdf

- Table2.pdf

- Table3.pdf

- Table4.pdf 